\title{
Design and Performance of an Electrically Small Antenna with Matching Circuit
}

\author{
H. Kanaya, R. Nabeshima, R. K. Pokharel, and K. Yoshida \\ Graduate School of Information Science and Electrical Engineering \\ Kyushu University \\ 744, Motooka, Fukuoka, 819-0395, JAPAN \\ kanaya@ed.kyushu-u.ac.jp
}

\begin{abstract}
We designed and fabricated an electrically small antenna (ESA) with coplanar waveguide (CPW) matching circuit. Matching circuit is realized by using an interdigital gap and a transmission line. We designed ESA with the aid of the commercial three-dimensional electro magnetic field simulator. We also made experiments on the ESA with CPW matching circuits using patterned circuit board.
\end{abstract}

Keywords-Slot antenna, electrically small antenna, coplanaer waveguide, matching circuit.

\section{INTRODUCTION}

Studies are made of an electrically small antenna (ESA), i.e., the antenna whose dimension is much smaller than onewavelength, towards further reduction of the antenna size in order to realize ubiquitous wireless systems $[1,2]$. It is widely known, however, that in order to realize the miniaturized antenna. Moreover, we must simultaneously realize a broadband impedance matching circuit, which compensates the narrow bandwidth peculiar to the small antenna with low radiation resistance, and we must attain large impedancematching ratios to connect with semiconductor amplifiers with high internal impedances. Also, the small antenna is sensitive to the conductor resistance because of its low radiation resistance, and the decrease of the radiation efficiency often makes serious problem.

Slot antennas with coplanar waveguide (CPW) feed line are preferable for monolithic microwave integrated circuit (MMIC's) and RFIC (radio frequency integrated circuit) since no via holes are required for integration with devices [3-5].

In our previous works, we designed a one-wavelength slot dipole antenna and a half-wavelength slot loop antenna with a bandpass filter using CPW lines, which acts as an impedance matching circuit as well [6-9]. Also, we have designed a slot dipole antenna whose dimension is much smaller than one wavelength with aid of EM-simulator, and carried out the experiments using high temperature superconductors YBCO thin film on the $\mathrm{MgO}$ substrate with relative permittivity of 9.6 in the $5.0 \mathrm{GHz}$ band [10].

In this paper, we design the impedance matching circuit, which connects an ESA fabricated on the normal metal to a semiconductor amplifier. The proposed matching circuits have performances similar to those of the $n$-pole bandpass filter (BPF) [11]. By using the quarter wavelength transmission line and admittance $(J)$-inverter, we can reduce the total antenna size more than one-wavelength. In the beginning, theoretical

This work was partly supported by a Grant-in-Aid for Scientific Research (C) from the Japan Society for the Promotion of Science (JSPS). performances of the ESA with the CPW impedance matching circuit are calculated by using the transmission line model as well as the commercial electro-magnetic (EM) field simulator (Ansoft; HFSS ver.10). In order to demonstrate the theory, we also carried out experiments on the ESA at $2.4 \mathrm{GHz}$, which has the size of $22.3 \mathrm{~mm} \times 8.204 \mathrm{~mm}$, with the pole number of $n=1$ matching circuits using FR4 printed circuit board.

\section{Design of THE ElECTRICAlly SMALl ANTENNA (ESA)}

The conventional slot antenna is a one-wavelength center feed slot antenna as shown in Fig. 1. The designed center frequency is $2.4 \mathrm{GHz}$-band. The substrate has dielectric constant $\varepsilon_{\mathrm{r}}=4.25$ and $\tan \delta=0.015$. The thickness of the substrate and copper top metal is $0.8 \mathrm{~mm}$ and $18 \mu \mathrm{m}$, respectively. Three-dimensional EM simulator simulates the RF properties. Fig. 2 shows the return loss of the standard slot antenna. The input impedance is almost $50 \Omega$ around $2.4 \mathrm{GHz}$, and $-10 \mathrm{~dB}$ bandwidth is $165 \mathrm{MHz}$

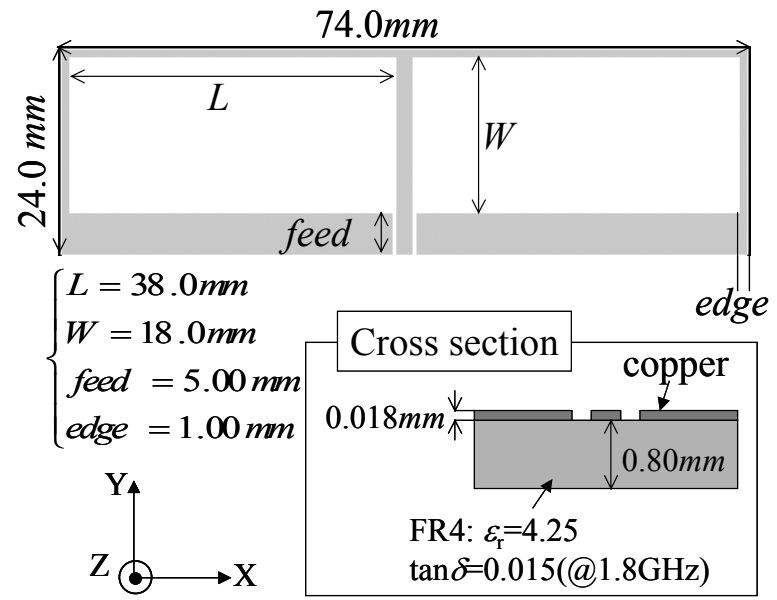

Figure 1. Layout of the standard slot dipole antenna.

Fig. 3 shows the simulated layout of an electrically small antenna (ESA). The physical properties of the substrate are the same as those of standard antenna. The antenna size is $0.18 \lambda_{0}$ and $0.067 \lambda_{0}$, respectively, where $\lambda_{0}$ is the wavelength in the vacuum at $2.45 \mathrm{GHz}$, which is especially smaller than that of the standard dipole slot antenna. Fig. 4 shows the $Z_{a}$ 
$\left(Z_{a}=R_{a}+j X_{a}\right)$ of the slot antenna, where $R_{a}$ represents radiation resistance and metal loss, and $X_{a}$ is the reactance of the antenna. Because the quarter-wavelength parallel resonance appears at $2.74 \mathrm{GHz}$, the size of this antenna is electrically smaller than quarter wavelength when the antenna works at $2.4 \mathrm{GHz} . Z_{a}$ is $42.5+j 385 \Omega$ at $2.4 \mathrm{GHz}$, which is far from $50 \Omega$, so that, it is shown that most RF signals reflect for the impedance missmatch.

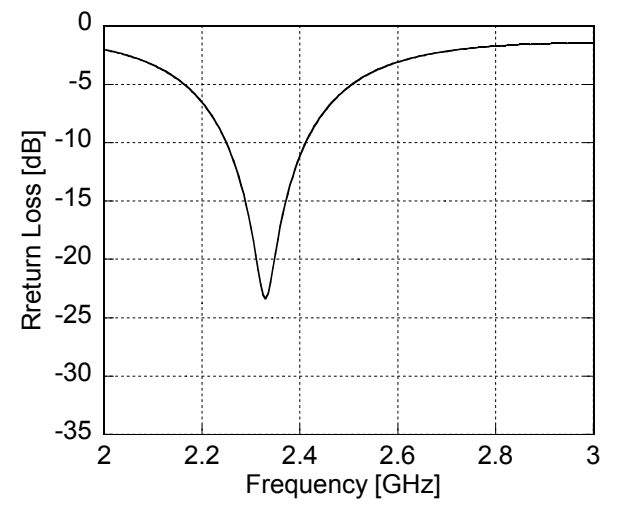

Figure 2. Return loss of the standard slot dipole antenna.

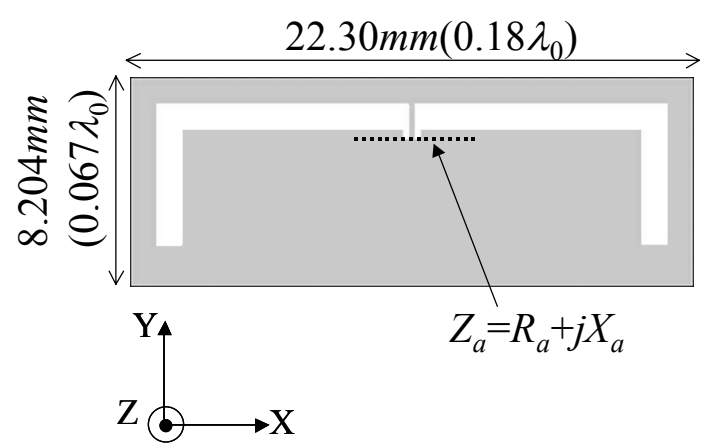

Figure 3. Layout of the electrically small slot dipole antenna.

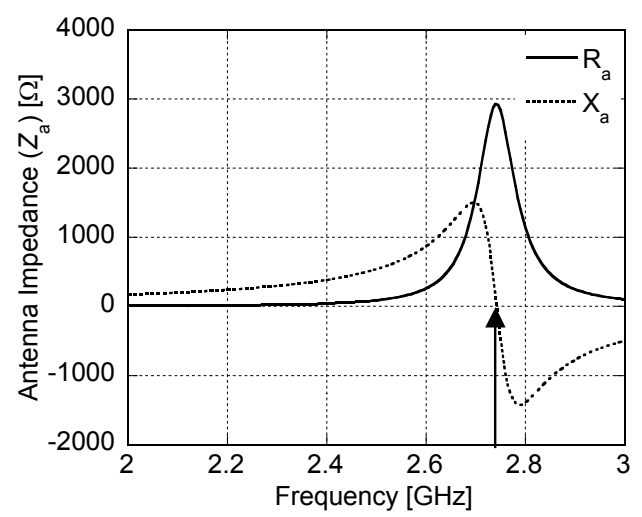

Figure 4. Input impedance of the ESA without matching circuit.

\section{DESIGN OF THE IMPEDANCE MATCHING CiRCUIT BETWEEN ESA AND SEMICONDUCTOR AMPLIFIER}

In order to connect between ESA and RF front-end, impedance matching must be attained between the antenna and amplifiers.
The present matching circuit is based on the bandpass filter (BPF) composed of the transmission line and $J$-inverters. Fig. 5(a) shows the $n=1 \mathrm{BPF}$, where, $Y_{0}$ is the admittance of the load and $B_{1}$ is the susceptance in the parallel resonator with a susceptance slope parameter $b_{1}$. The design parameters of the $n=1$ BPF are

$$
J_{01}=\sqrt{w} \sqrt{\frac{Y_{0} b_{1}}{g_{0} g_{1}}}, \quad J_{12}=\sqrt{w} \sqrt{\frac{b_{1} Y_{0}}{g_{1} g_{2}}}
$$

where, $w$ is the relative bandwidth and $g_{i}$ is the filter parameter. Fig. 5(b) shows the equivalent circuit model of the Fig. 5(a) at the center frequency. In the figure, $G_{S}{ }_{S}$ and $G^{\prime}{ }_{L}$, and $Q_{e i}$ are the equivalent conductance and external quality factor, respectively.

Fig. 6(a) shows the equivalent circuit model for the smallest matching circuit, corresponding to BPF with $n=1$. In this figure, $Z_{a}\left(=1 / Y_{a}\right)$ denotes the input impedance of ESA and $Z_{L}$ $\left(=1 / Y_{L}\right)$ represents the input impedance of LNA or the output impedance of PA. $Z_{01}\left(=1 / Y_{01}\right)$ and $\theta_{i}$ are characteristic impedance and electrical length of the transition line. Fig. 6(b) shows the equivalent circuit model of the Fig. 6(a) at the center frequency. In Fig. 6(a), $Y^{\prime}$ is the admittance for looking the antenna from $A-A^{\prime}$, and $Y^{\prime}{ }_{i n}$ is the admittance for looking amplifier from $A-A$ ' and given by,

$$
\begin{gathered}
Y^{\prime}=G^{\prime}+j B^{\prime}=Y_{01} \frac{Y_{a}+j Y_{01} \tan \theta_{01}}{Y_{01}+j Y_{a} \tan \theta_{01}} \\
Y_{i n}{ }^{\prime}=G_{i n}{ }^{\prime}+j B_{i n}{ }^{\prime}=\frac{\frac{1}{Z_{L}+1 / j \omega C_{m}}+j Y_{01} \tan \left(-\theta_{02}\right)}{Y_{01}+j \frac{1}{Z_{L}+1 / j \omega C_{m}} \tan \left(-\theta_{02}\right)}
\end{gathered}
$$

Finally, the comparison of the Fig. 5(b) and 6(b), the proposed design value $\theta_{01}, Z_{01}, \theta_{02}, C_{m}$ are led in the numerical value as follows:

$$
\begin{gathered}
\left.\operatorname{Im} B^{\prime}\right|_{\omega=\omega_{0}}=\left.\operatorname{Im} B_{i n}{ }^{\prime}\right|_{\omega=\omega_{0}}=0 \quad\left(\theta_{01}, \theta_{02} \leq \pi / 2\right) \\
\frac{b^{\prime}}{G^{\prime}}=Q_{e 1}\left(=\frac{g_{0} g_{1}}{w}\right) \\
\frac{b^{\prime}}{G_{i n}{ }^{\prime}}=Q_{e 2}\left(=\frac{g_{1} g_{2}}{w}\right) \\
b^{\prime}=\left.\frac{\omega_{0}}{2} \frac{\partial B^{\prime}\left(Z_{01}, \theta_{01}\right)}{\partial \omega}\right|_{\omega=\omega_{0}} .
\end{gathered}
$$

Fig. 7 shows the simulated layout of the ESA with CPW transmission line which has $Z_{01}$ and $\theta_{01}$ (See $A$ - $A^{\prime}$ in Fig. 6 (a)). Fig. 8 shows the frequency dependence of the $Y$ '. We can see the parallel resonance around $2.4 \mathrm{GHz}$.

Fig. 9 shows the layout of ESA with CPW matching circuit. In order to realize $\theta_{02}$ and $C_{m}$, we adopted the interdigital gap and CPW transmission line, where $Z_{L}$ is assumed to be $Z_{0}=50$ 
$\Omega$ by the experiment to be convenient. Fig. 10 shows the input impedance $\left(Z_{i n}\right)$, which is the impedance for looking the antenna from $B-B$ ' in Fig. 6 and Fig. 9. Fig. 11 shows the return loss and Smith chart of the ESA with CPW matching circuit. $Z_{\text {in }}$ is almost $50 \Omega$ around $2.4 \mathrm{GHz}$, so that, return loss is $-32.58 \mathrm{~dB}$ at $2.4 \mathrm{GHz}$. Fig. 12 shows the simulated radiation pattern of the ESA with CPW matching circuit.

(a)

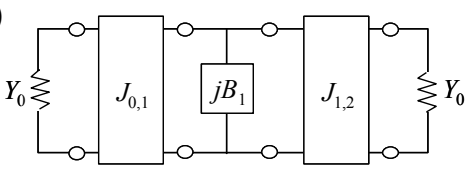

(b)

$$
G_{s}^{\prime}=\frac{b_{1}}{Q_{e 1}} \xi \frac{j}{\mathrm{j} B_{1}} \xi G_{L}^{\prime}=\frac{b_{2}}{Q_{e 2}}
$$

Figure 5. Circuit model of the $n=1$ bandpass filter (a) and equivalent circuit model at center frequency (b).

(a)

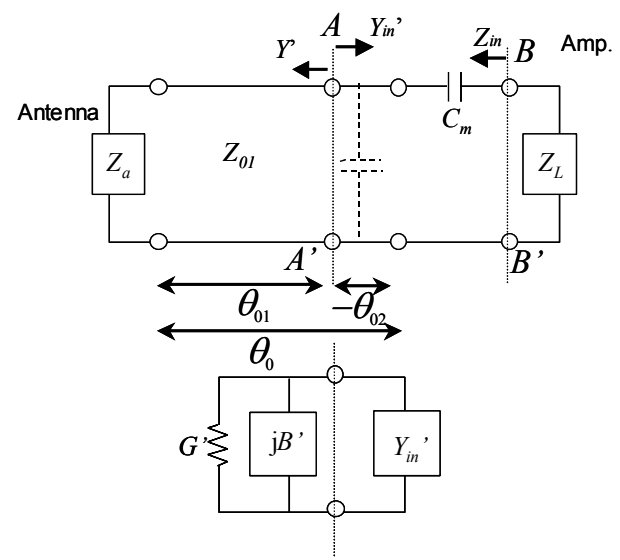

Figure 6. Circuit model of the $n=1$ impedance matching circuit (a) and equivalent circuit model at center frequency (b).

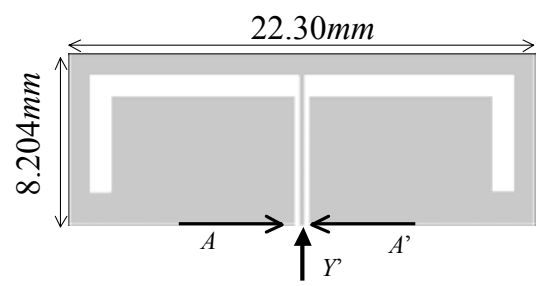

Figure 7. Layout of the electrically small antenna with CPW feed line.

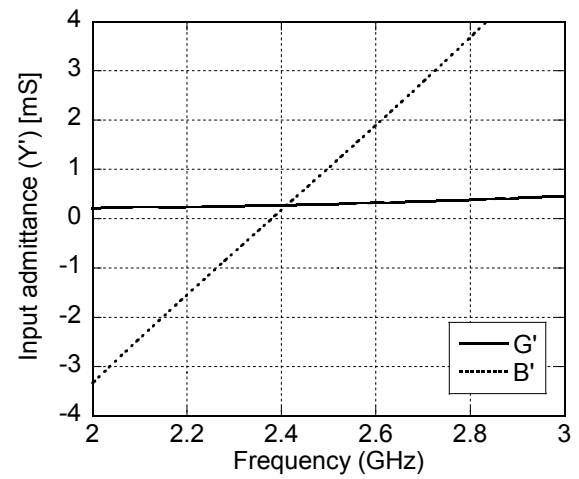

Figure 8. Input admittance $\left(Y^{\prime}\right)$ of the ESA with $\mathrm{CPW}$ transmission line.

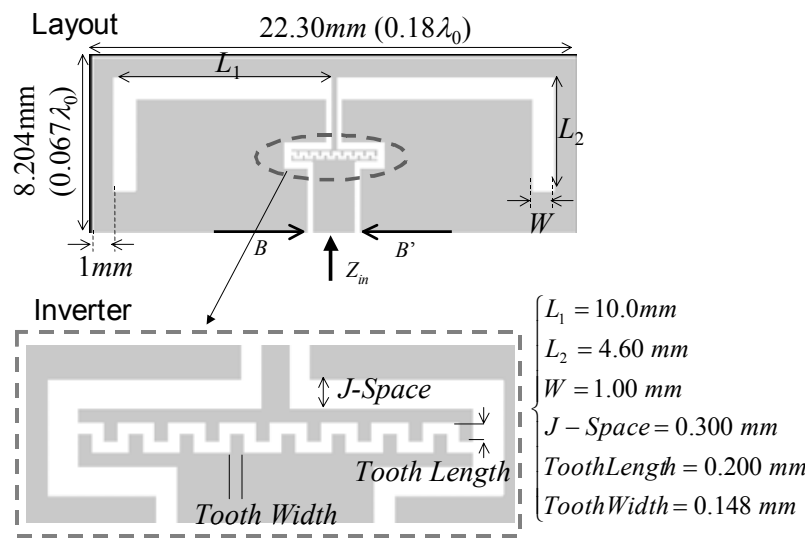

Figure 9. Layout of the ESA with CPW matching circuit.

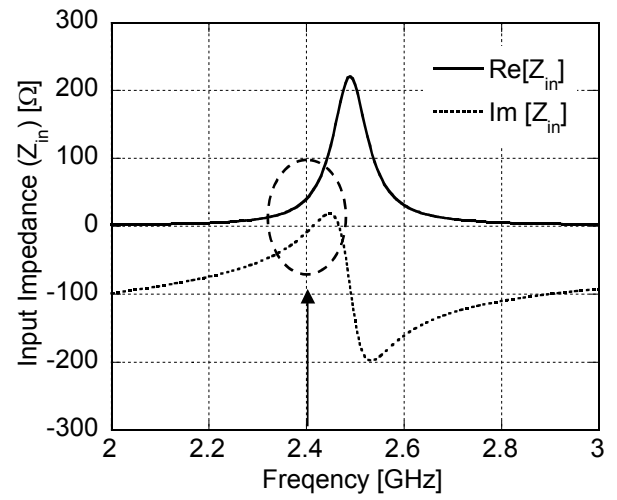

Figure 10. Input impedance of the ESA with matching circuit.
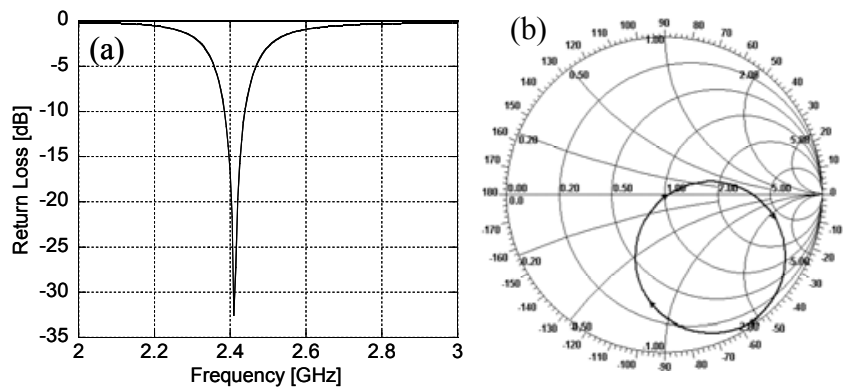

Figure 11. Return loss (a) and Smith chart (b) of the ESA with CPW matching circuit.

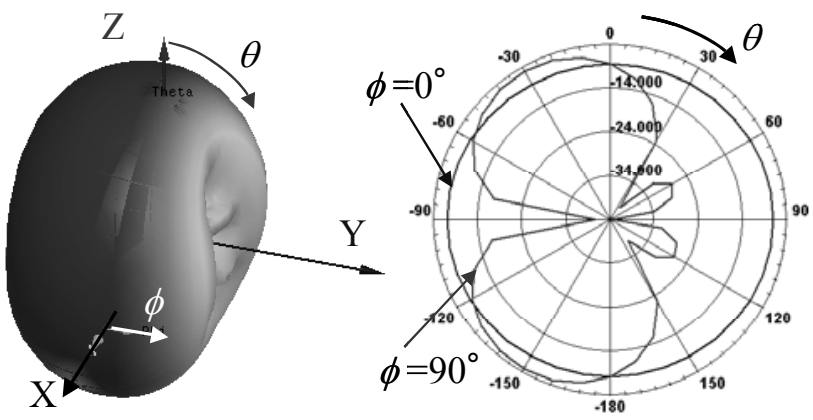

Figure 12. Simulated radiation pattern of the ESA. 


\section{EXPERIMENTAL RESULTS AND DISCUSSIONS}

An ESA is fabricated on FR4 substrate by using the print board making equipment (MITS; FP-21T model 40), which has $100 \mu \mathrm{m}$-diameter milling cutter. Fig. 13 shows the photographs of the ESA with CPW matching circuit. In the figure, an interdigital gap is also shown. RF signal is input through MMCX connecter, which has characteristic impedance $=50 \Omega$. We measured the $S$-parameters by using a GP-IB controlled vector network analyzer (HP; HP8722C).

Fig. 14 shows the experimental results of the return loss of the ESA with CPW matching circuit. The observed experimental results are caused by an error in edge part of the interdigital gap (see Fig. 13), an error of the dielectric constant of the FR4 substrate and residual loss of the connection between connector and antenna. So, simulated results taking account of the permittivity error are also shown. In EM simulation, we made the $\varepsilon_{\mathrm{r}}$ from 4.25 to 4.05 , so that, the experiment results and the simulation values are corresponding well.

Fig. 15 shows the comparison of the standard slot antenna with the ESA. The antenna size can be reduced to about $90 \%$ and bandwidth design becomes possible.

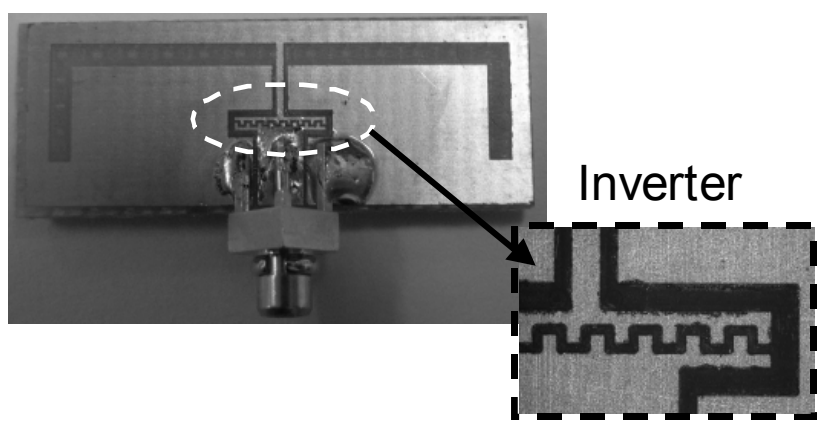

Figure 13. Photograph of the ESA.

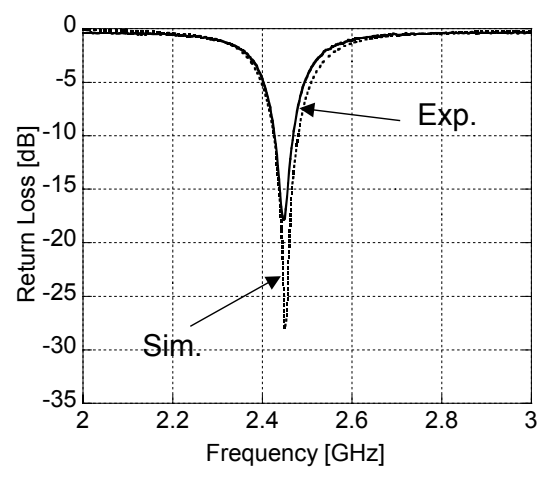

Figure 14. Experimental results and simulated results by taking account of permittivity error.

\section{CONCLUSIONS}

In this paper, slot dipole antenna with a bandpass filter have been designed and tested. We succeeded in realizing the circuit which matches the small radiation resistance of ESA to the amplifier. As a result, we designed a small-size $(22.30 \mathrm{~mm}$ x $8.204 \mathrm{~mm}$ ) slot antenna. Moreover, ESA with CPW matching circuit was fabricated and measured the RF properties, thereby we demonstrated frequency characteristics as expected.

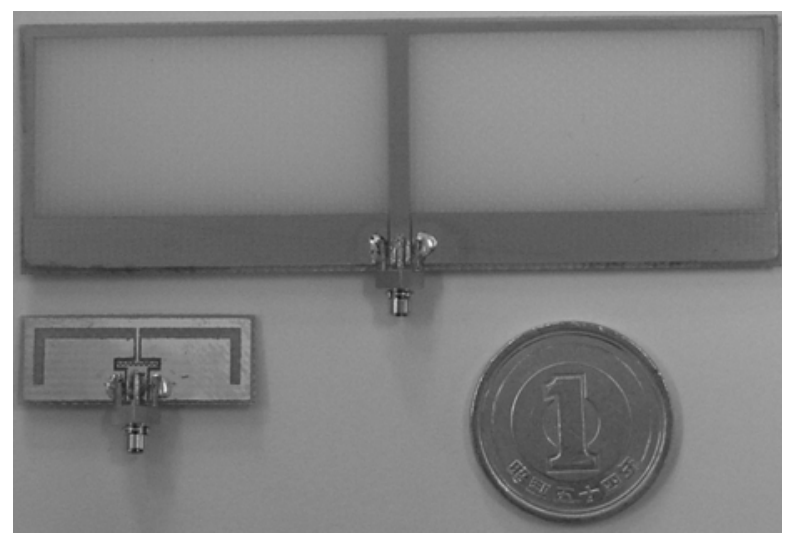

Figure 15. Comparison of the standard slot antenna with the ESA.

\section{REFERENCES}

[1] N. Behdad, and K. Sarabandi, "Bandwidth enhancement and further size reduction of a class of miniaturized slot antennas", IEEE Trans. Antennas Propagat., vol. 52, no. 8, pp. 1928-1935, August 2004.

[2] R. Azadegan and K. Sarabandi, "A Novel Approach for Miniaturization of Slot Antennas," IEEE Trans. Antennas Propagat., vol. 51, no. 3, pp. 421-429, March 2003

[3] B.K. Kormanyos, W. Harokopus, L. Katehi, and G. Rebeiz, "CPW-fed active slot antennas", IEEE Trans. Microwave Theory \& Thech., vol. 42, pp.541-545, April, 1994.

[4] S. S. Garcia, and J. J. Laurin, " Study of a CPW Inductively coupled slot antenna", IEEE Trans. Antennas Propagat., vol. 47, no. 1, pp. 58-64, January 1999.

[5] A. U. Bhobe, C. L. Holloway, M. P. May, and R. Hall, "Wide-band slot antenna with CPW fed lines: Hybrid and log-periodic designs", IEEE Trans. Antennas Propagat., vol. 52, no. 10, pp. 2545-2554, October 2004.

[6] K. Yoshida, T. Takahashi, H. Kanaya, T. Uchiyama, and Z. Wang, "Superconducting slot antenna with broadband impedance matching circuit," IEEE Trans. Appl. Supercond., vol. 11, pp. 103-106, March 2001.

[7] H. Kanaya, Y. Koga, J. Fujiyama, G. Urakawa and K. Yoshida, "Design and Performance of High Tc Superconducting Coplanar Waveguide Matching Circuit for RF-CMOS LNA," IEICE Trans. Electron., vol. E86-C, no. 1, pp. 37-41, January 2003.

[8] H. Kanaya, Y. Koga, G. Urakawa, and K. Yoshida, "Design of HTS Coplanar Waveguide Matching Circuit for Low Noise CMOS-HTS Receiver," IEEE Trans. on Appl. Supercond., vol. 13, no. 2, pp. 10311034, June 2003.

[9] K. Yoshida, Y. Tsutsumi, and H. Kanaya "Electrically Small Antennas with Miniaturized Impedance Matching Circuits for Semiconductor Amplifiers," IEICE Trans. Electron., vol. E88-C, pp1368-1374, no. 7, July 2005.

[10] S. Oda, S. Sakaguchi, H. Kanaya, R.K. Pokharel, and K. Yoshida, "Electrically Small Superconducting Antennas with Bandpass Filters", IEEE Trans. on Appl. Supercond., vol. 17, 2007 (in press).

[11] G. L. Matthaei, L. Young and E. M. T. Jones, Microwave Filters, Impedance-Matching Networks, and Coupling Structures, Norwood, MA: Artech House. 1980. 